\title{
Advantages of the Blue InGaN/GaN Light-Emitting Diodes with an AlGaN/GaN/AIGaN Quantum Well Structured Electron Blocking Layer
}

\author{
Zhen Gang Ju, ${ }^{\dagger}$ Wei Liu, ${ }^{\dagger}$ Zi-Hui Zhang, ${ }^{\dagger}$ Swee Tiam Tan, ${ }^{\dagger}$ Yun Ji, ${ }^{\dagger}$ Zabu Kyaw, ${ }^{\dagger}$ Xue Liang Zhang, \\ Shun Peng Lu, ${ }^{\dagger}$ Yi Ping Zhang, ${ }^{\dagger}$ Bin Bin Zhu, ${ }^{\dagger, \dagger}$ Namig Hasanov, ${ }^{\dagger}$ Xiao Wei Sun, ${ }^{*},{ }^{\dagger}$ \\ and Hilmi Volkan Demir*,†, $* \$$ \\ ${ }^{\dagger}$ LUMINOUS! Centre of Excellence for Semiconductor Lighting and Displays, School of Electrical and Electronic Engineering, \\ Nanyang Technological University, 50 Nanyang Avenue, Singapore, 639798 \\ ${ }^{\sharp}$ School of Physical and Mathematical Sciences, Nanyang Technological University, 21 Nanyang Link, Singapore, 637371 \\ ${ }^{\S}$ Department of Electrical and Electronics Engineering, Department of Physics, and UNAM-Institute of Materials Science and \\ Nanotechnology, Bilkent University, TR 06800, Ankara, Turkey
}

\section{Supporting Information}

ABSTRACT: InGaN/GaN light-emitting diodes (LEDs) with $\mathrm{p}-(\mathrm{AlGaN} / \mathrm{GaN} / \mathrm{AlGaN})$ quantum well structured electron blocking layer (QWEBL) are designed and grown by a metalorganic chemical-vapor deposition (MOCVD) system. The proposed QWEBL LED structure, in which a p-GaN QW layer is inserted in the $\mathrm{p}-\mathrm{AlGaN}$ electron blocking layer, not only leads to an improved hole injection but also reduces the electron leakage, thus enhancing the radiative recombination rates across the active region. Consequently, the light output power was enhanced by $10 \%$ for the QWEBL LED at a current density of $35 \mathrm{~A} / \mathrm{cm}^{2}$. The efficiency droop of the optimized device was reduced to $16 \%$. This is much smaller than that of the conventional p-AlGaN electron blocking layer LED, which is $31 \%$.

KEYWORDS: GaN, LED, QW, EBL, MOCVD

\begin{abstract}
— or InGaN/GaN light-emitting diodes (LEDs) working as - the light source for artificial lighting, a high operating current density is necessary to generate sufficient light output power. However, at high current densities an efficiency droop is often observed, and many efforts have been made to suppress the efficiency droop. ${ }^{1}$ A p-AlGaN electron blocking layer (EBL), as one of the basic LED structure layers, is typically applied to fulfill this function. However, it has been found that although the p-AlGaN EBL is able to reduce the overflow of the electrons from the active region to the $\mathrm{p}-\mathrm{GaN}$ layer, it also retards the hole injection from the $\mathrm{p}-\mathrm{GaN}$ layer to the active region. ${ }^{2}$ Many variants based on the p-AlGaN EBL have been proposed and realized, which have greatly enhanced the optical power and reduced the efficiency droop under high current intensity. These approaches include p-AlGaN EBLwith graded Al composition, ${ }^{3}$ AlGaN/GaN superlattice EBL, ${ }^{4}$ AlInGaN quaternary EBL, ${ }^{5}$ and AlInN EBL. ${ }^{6}$ In practice, the graded $\mathrm{Al}$ composition EBL and the superlattice EBL are not easy to be realized since they need very precise control of the composition and the thickness of the EBL layer. ${ }^{7}$ For quaternary AlInGaNand AlInN-type EBLs, due to the large difference of AlN and $\mathrm{InN}$ in bonding strength and thermal property, the growth has to be conducted at a low temperature with $\mathrm{N}_{2}$ as carrier gas. Therefore, the growth conditions are difficult to control and the material quality is often compromised. Moreover, the degraded
\end{abstract}

quality of the EBL will also lead to the degradation of the quality of the subsequent p-GaN layer. ${ }^{8}$ To simplify the growth process, guarantee a high crystal quality, and maintain the electron blocking function of the EBL, Xia et al. have theoretically demonstrated the advantages of the p-type $\mathrm{AlGaN} / \mathrm{GaN} / \mathrm{AlGaN}$ (AGA) EBL with an inserted $\mathrm{p}-\mathrm{GaN}$ layer thicker than $4 \mathrm{~nm}^{9}$ However, there is no experimental study performed to prove their concept.

In this work, different from previously studied structures, we initiate a new type of AGA EBL with a thin p-GaN layer of thickness less than $4 \mathrm{~nm}$ : the thin $\mathrm{p}-\mathrm{GaN}$ layer serves as a quantum well $(\mathrm{QW})$, and the $\mathrm{AlGaN}$ layers with an $\mathrm{Al}$ composition of $20 \%$ were regarded as the barriers. Therefore, the proposed architecture is a quantum well structured EBL (QWEBL). The improvements in electron confinement and hole injection in the LED with the QWEBL are predicted by theoretical simulations and confirmed experimentally here. The designed LED structure with the optimized QW thickness of 2 $\mathrm{nm}$ not only suppresses the electron overflow of the active region but also enhances the hole injection. The efficiency droop in the LED with the proposed QWEBL structure is

Received: January 1, 2014

Published: March 21, 2014 
found to be much smaller than that in the LED with the conventional p-AlGaN EBL.

\section{EXPERIMENTAL METHODS}

InGaN/GaN LEDs studied in this work were grown by an Aixtron metal-organic chemical-vapor deposition (MOCVD) system. Two-inch patterned sapphire substrates with periodic cone patterns (with a diameter of $2.4 \mu \mathrm{m}$, a height of $1.5 \mu \mathrm{m}$, and a pitch of $3 \mu \mathrm{m}$ ) were used. The growth started with a 30 $\mathrm{nm}$ thick low-temperature $\mathrm{GaN}$ buffer, followed by an undoped-GaN interlayer ( $150 \mathrm{~nm}$ thick); detailed information on the interlayer growth can be found in our previous publication. ${ }^{10}$ Subsequently, a high-temperature undoped GaN was grown with a thickness of $5 \mu \mathrm{m}$ followed by a $3 \mu \mathrm{m} \mathrm{Si-}$ doped $\mathrm{n}-\mathrm{GaN}$ layer. Six pairs of InGaN/GaN multiple quantum wells (MQWs) were grown with the $3 \mathrm{~nm}$ quantum well at 750 ${ }^{\circ} \mathrm{C}$ and the $12 \mathrm{~nm}$ quantum barriers at $800{ }^{\circ} \mathrm{C}$. The indium composition of the InGaN well is $15 \%$, and the peak emission wavelength is $450 \mathrm{~nm}$. The p-EBL was grown on the top of the last barrier. The structures were finally covered with a $200 \mathrm{~nm}$ thick p-GaN layer. For the reference sample a p- $\mathrm{Al}_{0.2} \mathrm{Ga}_{0.8} \mathrm{~N}$ layer of $28 \mathrm{~nm}$ was used as the p-EBL. In the proposed QWEBL LED samples, the p-EBL was a p-(AlGaN/GaN/ AlGaN) structure with the total thickness kept at $28 \mathrm{~nm}$, and the thin $\mathrm{p}-\mathrm{GaN}$ layer thickness $(L)$ was chosen as being either 2.0 or $4.0 \mathrm{~nm}$. The QWEBL structure is simply realized by closing the TMAl valve during the $\mathrm{QW}$ growth of the AlGaN EBL. The growth temperature and pressure are kept at $980{ }^{\circ} \mathrm{C}$ and 100 mbar the entire time. The thickness and composition of each layer is precisely controlled with mole ratios and growth time based on the calibration runs. The detailed schematic structure is shown in Figure 1. The indium contacts on the epi-

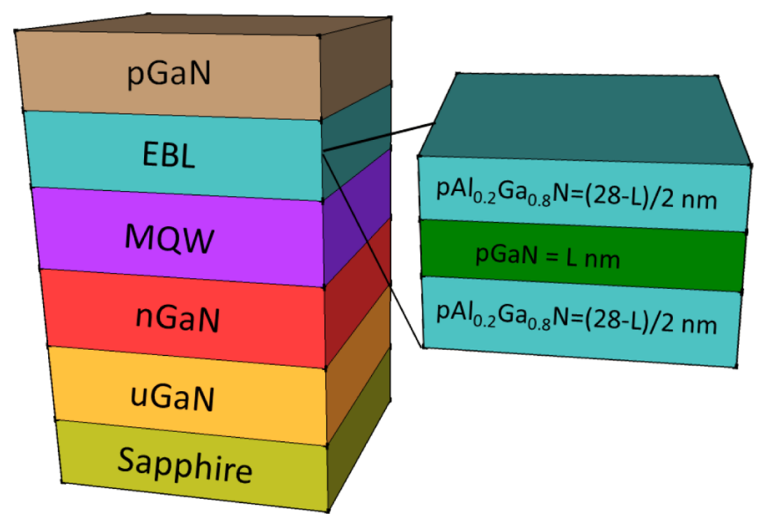

Figure 1. Schematic diagrams of the LEDs with the conventional EBL $(L=0)$ and QWEBL $(L=2$ and $4 \mathrm{~nm})$.

wafers were made in a circular area of $1.5 \mathrm{~mm}$ diameter for characterization purposes. The optical power was measured by an integrating sphere attached to an Ocean Optics spectrometer (QE65000), which was calibrated with a standard light source.

On the basis of our grown structures, numerical simulations were performed using APSYS to understand the working mechanisms of the proposed QWEBL structures. The simulator solves Schrödinger-Poisson equations self-consistently. The simulation has also taken the Coulomb interaction into consideration with the typical dielectric constants of IIInitrides. ${ }^{11}$ The simulation parameters including the Auger recombination coefficients, the Shockley-Read-Hall recombi- nation coefficient, the energy band offset ratio for the MQWs, and the polarization level for devices along the $c$-orientation can be found in our previous publication. ${ }^{12}$

\section{RESULTS AND DISCUSSION}

Figure $2 a-c$ show the simulated results of the hole concentration distribution in the quantum wells for the conventional EBL LED and the QWEBL LEDs at a current density level of $35 \mathrm{~A} / \mathrm{cm}^{2}$. As depicted in Figure $2 \mathrm{a}$ and $\mathrm{b}$, the hole concentration is much higher in each individual quantum well in the QWEBL LED with the p-GaN QW thickness of 2 $\mathrm{nm}$, compared to the conventional EBL LED. However, when the thickness of the p-GaN QW is increased to $4 \mathrm{~nm}$ as shown in Figure $2 c$, the hole concentration in the InGaN quantum wells becomes lower than that in the conventional EBL LED. Figure $2 \mathrm{~d}$ highlights the hole concentration in the EBL region. Figure 3 shows the electron concentration in the $\mathrm{p}-\mathrm{EBL}$ and the $\mathrm{p}-\mathrm{GaN}$ region. It can be seen that the two QWEBLs enable a lower electron leakage into the $\mathrm{p}-\mathrm{GaN}$ region, which indicates that the QWEBL allows for a better electron blocking effect than the conventional EBL. Moreover as shown in Figure 3, a higher electron concentration is observed in the p-EBL layer and in the $\mathrm{p}-\mathrm{GaN}$ region for the QWEBL LED with a $4 \mathrm{~nm}$ pGaN QW than that of the QWEBL LED with a $2 \mathrm{~nm}$ p-GaN QW, indicating an even better electron blocking effect of the latter. These results suggest that the electrons can be more effectively confined in the MQW region and that the hole injection efficiency can be improved remarkably by using the QWEBL with $L=2 \mathrm{~nm}$. As a result, the radiative recombination rate in the LED with QWEBL is improved significantly as shown in Figure 4 compared with the conventional EBL LED. However, the radiative recombination rates will drop if the GaN layer thickness is $4 \mathrm{~nm}$. This is due to the stronger hole confinement in the thicker GaN layer, as shown in Figure 2d.

Figure $5 \mathrm{a}-\mathrm{c}$ present the energy band diagrams of the LEDs with the conventional EBL and the QWEBL at $35 \mathrm{~A} / \mathrm{cm}^{2}$. As is well known, in conventional EBLs, due to the stronger polarization effect in the $\mathrm{QW}$ region, the effective barrier height of the EBL is reduced for electrons and increased for the holes, which lowers the electron blocking capability and hinders the injection efficiency of holes from the $\mathrm{p}-\mathrm{GaN}$ region into the MQWs. ${ }^{13}$ The combination of these effects results in a low hole concentration in the InGaN MQWs and a large electron leakage, as shown in Figures $2 \mathrm{a}$ and 3 . However, as the conventional EBL is replaced by the QWEBL, the effective barrier height is changed with the insertion of the $\mathrm{p}-\mathrm{GaN} \mathrm{QW}$ as shown in Figure 5. When the p-GaN QW is $2 \mathrm{~nm}$ thick, the effective barrier height for electrons is increased from $366 \mathrm{meV}$ to $416 \mathrm{meV}$ in the QWEBL LED, while its effective barrier height for holes is reduced from $469 \mathrm{meV}$ to $457 \mathrm{meV}$ as shown in Figure $5 a$ and $b$. These changes in the effective barrier heights for electrons and holes are the main cause of the electron overflow reduction and the hole injection enhancement. The constructive combinational effect of the electron overflow reduction and the hole injection enhancement leads to the enhancement of the radiative recombination rate as shown in Figure $4 \mathrm{~b}$. Nonetheless, the change in the effective barrier height is dependent on the thickness of the p-GaN QW. As the p-GaN QW thickness is increased to $4 \mathrm{~nm}$, although the effective barrier height for electrons is increased from $366 \mathrm{meV}$ to $411 \mathrm{meV}$ in the QWEBL LED, the effective barrier height for holes is also increased from $469 \mathrm{meV}$ to $479 \mathrm{meV}$ in the QWEBL LED, as shown in Figure 5a and c. The benefit of the 

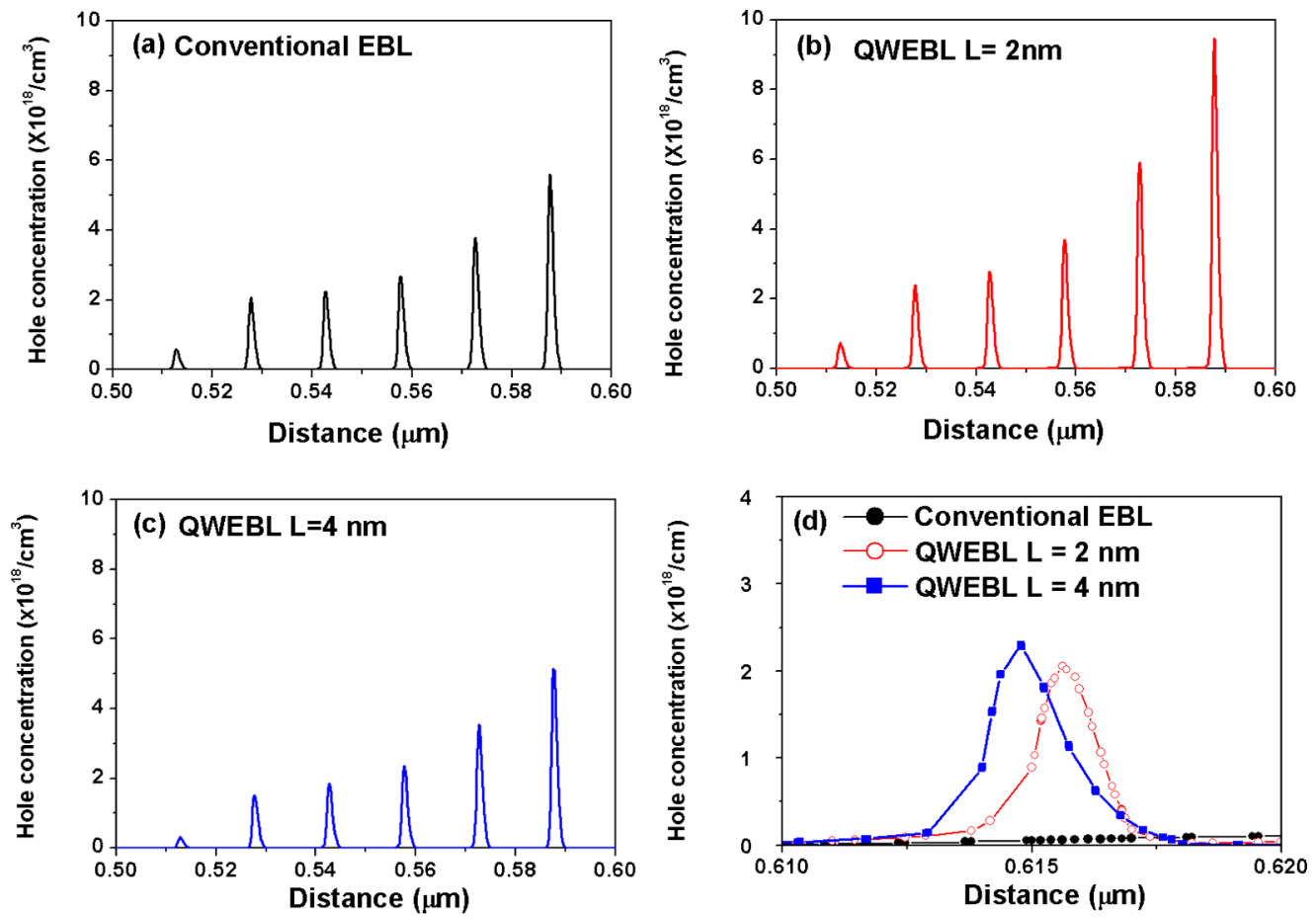

Figure 2. Simulated $(a-c)$ hole concentrations of QWs for the conventional EBL LED and the QWEBL LED at the current injection level of 35 A/ $\mathrm{cm}^{2}$. (d) Hole concentrations within the EBL region for the conventional EBL LED and the QWEBL LED at the current injection level of $35 \mathrm{~A} / \mathrm{cm}^{2}$.

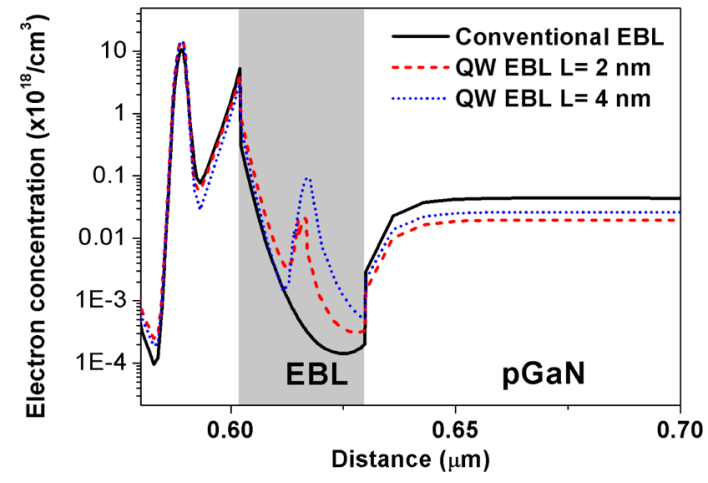

Figure 3. Electron concentration in EBL and $p-G a N$ for the conventional EBL LED and the QWEBL LED at the current injection level of $35 \mathrm{~A} / \mathrm{cm}^{2}$.

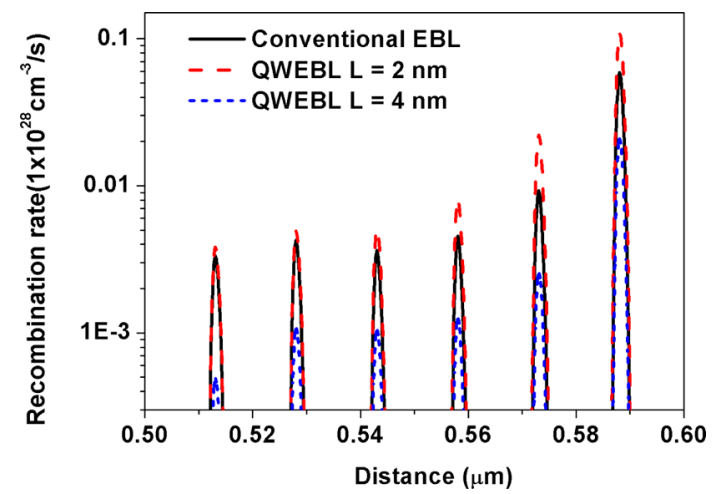

Figure 4. Radiative recombination rates at $35 \mathrm{~A} / \mathrm{cm}^{2}$ for the conventional EBL LED and the QWEBL LED.

electron overflow reduction due to the increase of the effective barrier height for electrons is offset by the reduction of hole injection efficiency due to the increased effective barrier height for holes. This leads to the lower radiative recombination rate as shown in Figure 4c. The reasons for the $\mathrm{p}-\mathrm{GaN} \mathrm{QW}$ thickness dependence of the effective barrier heights of the QWEBL LED are related to the formation of quantized states in the p-GaN QW and carrier tunneling process and will be discussed separately in detail in another publication.

The experimentally measured external quantum efficiency (EQE) and the optical power as a function of the current density are depicted in Figure $6 \mathrm{a}$ and $\mathrm{b}$ for all the devices. Compared to the conventional EBL LED, the best performance is observed from the QWEBL LED with the p-GaN QW of $L=$ $2 \mathrm{~nm}$. When we evaluate the performance of the QWEBL LED and the conventional EBL LED at the current density of $35 \mathrm{~A} /$ $\mathrm{cm}^{2}$, the optical output power of the QWEBL LED is $585 \mathrm{~mW}$, which is about $10 \%$ higher than that of the conventional EBL LED $(530 \mathrm{~mW})$. The efficiency droop of the QWEBL LED at $35 \mathrm{~A} / \mathrm{cm}^{2}$ is only $16 \%$. This is much smaller than that of the conventional EBL LED, which is $31 \%$. The improvement of the optical power and the EQE is well attributed to the improved hole injection and electron blocking in the QWEBL LED, as demonstrated in Figures 2 and 3. However, the optical output power of the QWEBL LED with $L=4 \mathrm{~nm}$ is even a little bit lower than the conventional EBL LED at $35 \mathrm{~A} / \mathrm{cm}^{2}$. This is consistent with the theoretical simulation results shown in Figures 2 and 3. Our results given above are quite different from the prediction of Xia et al., while suggesting that a thicker $\mathrm{GaN}$ layer leads to better performance. We believe that the thicker $\mathrm{p}-\mathrm{GaN}$ QW layer will trap more holes in the EBL region due to the low mobility of holes, as shown in Figure $2 \mathrm{~d}$. Meanwhile, the effect of the electron blocking will be reduced due to the thinner effective thickness of the AlGaN layer, as shown in Figure 3. Therefore, the thinner $\mathrm{p}-\mathrm{GaN} \mathrm{QW}$ in the EBL layer is favorable not only for blocking the electrons but also for easier transportation of holes from the p-type GaN 

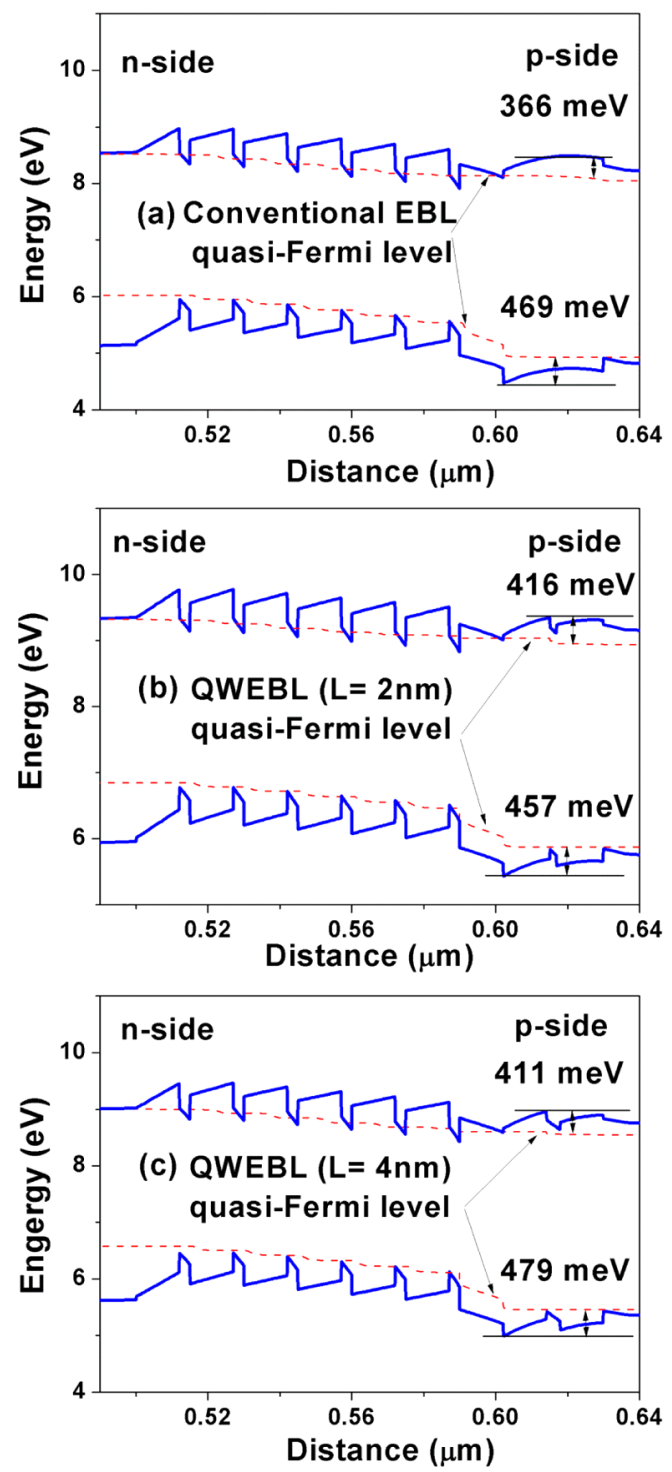

Figure 5. $(a-c)$ Energy band diagrams of the LEDs with the conventional EBL and the QWEBL at the current injection level of 35 $\mathrm{A} / \mathrm{cm}^{2}$.

region to the $\mathrm{p}-\mathrm{GaN} \mathrm{QW}$ of the QWEBL and finally to the MQWs region due to the tunneling effect. It should be noted that the conventional EBL LED shows a better performance at very low current injection level. This could be due to the fact that the initial hole confinement in the QW at low current levels may affect the hole injection efficiency for the QW EBL LED. As the hole tunneling effect increases rapidly with increasing current due to the field-assistance and resonance effects, the hole injection efficiency of the QW EBL LED will surpass that of the conventional EBL LED. The high hole injection efficiency combined with the high electron blocking effect will lead to a better performance at high current levels for the QW EBL LED.

\section{CONCLUSIONS}

In conclusion, blue InGaN/GaN MQW LEDs with the conventional p-AlGaN EBL and the p-(AlGaN/GaN/AlGaN) QWEBL have been investigated both numerically and experimentally. When the QWEBL is adopted, the quantum states are created, which results in the increase of the effective
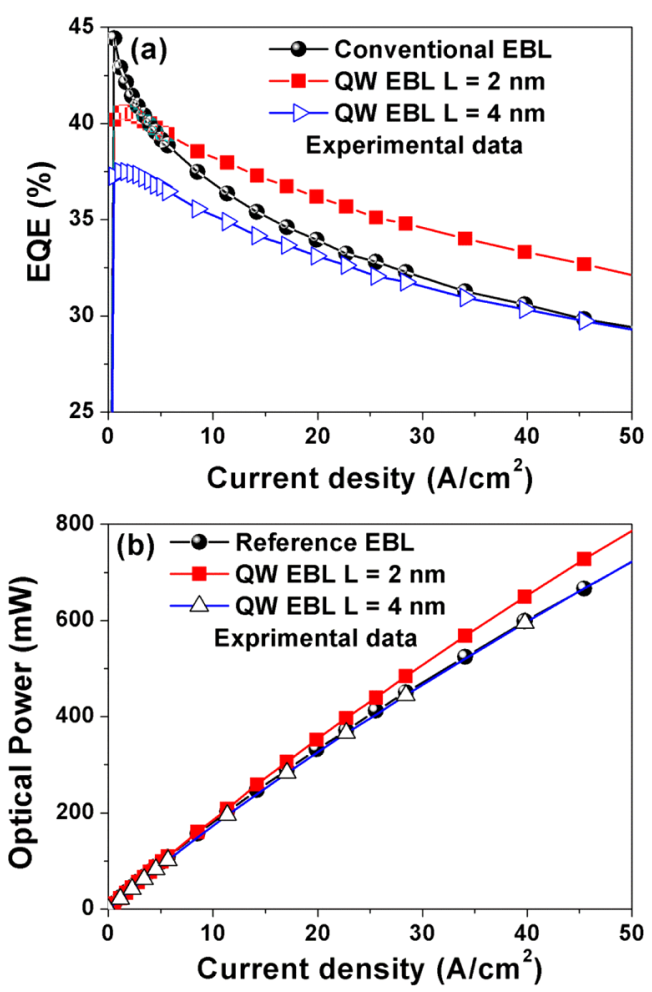

Figure 6. Experimentally measured (a) EQE and (b) optical power output with increasing current density for the conventional EBL and QWEBL LEDs.

barrier height for the electrons and the decrease of that for the holes. Moreover, with the well-controlled thickness of the p$\mathrm{GaN}$, more holes can tunnel into the MQWs from the p-type $\mathrm{GaN}$ region. These effects prevent electron leakage into the $\mathrm{p}-$ $\mathrm{GaN}$ region and improve the hole injection into the MQW region, which enables the improved optical performance in the LEDs with the proposed QWEBL.

\section{ASSOCIATED CONTENT}

\section{Supporting Information}

The details of the measurement for the optical power, XRD, EL information, and simulation data of hole concentration in the last QW and EBL. This information is available free of charge via the Internet at http://pubs.acs.org.

\section{AUTHOR INFORMATION}

\section{Corresponding Authors}

*E-mail: exwsun@ntu.edu.sg.

*E-mail: volkan@stanfordalumni.org.

\section{Notes}

The authors declare no competing financial interest.

\section{ACKNOWLEDGMENTS}

This work is supported by the National Research Foundation of Singapore under Grant Nos. NRF-CRP-6-2010-2 and NRF-RF2009-09 and the Singapore Agency for Science, Technology and Research (A*STAR) SERC under Grant No. 112120 2009.

\section{REFERENCES}

(1) Tan, S. T.; Sun, X. W.; Demir, H. V.; DenBaars, S. P. Advances in the LED materials and architectures for energy-saving solid-state 
lighting toward "lighting revolution". IEEE Photonics J. 2012, 4, 613619.

(2) Kim, M. H.; Schubert, M. F.; Dai, Q.; Kim, J. K.; Schubert, E. F.; Piprek, J.; Park, Y. Origin of efficiency droop in GaN-based lightemitting diodes. Appl. Phys. Lett. 2007, 91, 183507-3.

(3) Zhang, N.; Liu, Z.; Wei, T. B.; Zhang, L.; Wei, X. C.; Wang, X. D.; Lu, H. X.; Li, J. M.; Wang, J. X. Effect of the graded electron blocking layer on the emission properties of $\mathrm{GaN}$-based green lightemitting diodes. Appl. Phys. Lett. 2012, 100, 053504-3.

(4) Zhang, Y. Y.; Yin, Y. A. Performance enhancement of blue lightemitting diodes with a special designed $\mathrm{AlGaN} / \mathrm{GaN}$ superlattice electron-blocking layer. Appl. Phys. Lett. 2011, 99, 221103-3.

(5) Kim, S. J.; Son, S. H.; Kim, T. G. Improved light output power in GaN-based vertical light-emitting diodes with p-AlInGaN/GaN superlattices. J. Korean Phys. Soc. 2012, 60, 1258-1262.

(6) Choi, S.; Kim, H. J.; Kim, S.-S.; Liu, J.; Kim, J.; Ryou, J.-H.; Dupuis, R. D.; Fischer, A. M.; Ponce, F. A. Improvement of peak quantum efficiency and efficiency droop in III-nitride visible lightemitting diodes with an InAlN electron-blocking layer. Appl. Phys. Lett. 2010, 96, 221105-3.

(7) Chang, S. J.; Yu, S. F.; Lin, R. M.; Li, S. G.; Chiang, T. H.; Chang, S. P.; Chen, C. H. InGaN-based light-emitting diodes with an AlGaN staircase electron blocking layer. IEEE Photonics Technol. Lett. 2012, 24, 1737-1740.

(8) Wang, C. H.; Ke, C. C.; Lee, C. Y.; Chang, S. P.; Chang, W. T.; Li, J. C.; Li, Z. Y.; Yang, H. C.; Kuo, H. C.; Lu, T. C.; Wang, S. C. Hole injection and efficiency droop improvement in InGaN/GaN lightemitting diodes by band-engineered electron blocking layer. Appl. Phys. Lett. 2010, 97, 261103-3.

(9) Xia, C. S.; Li, Z. M. S.; Lu, W.; Zhang, Z. H.; Sheng, Y.; Da Hu, W.; Cheng, L. W. Efficiency enhancement of blue InGaN/GaN lightemitting diodes with an $\mathrm{AlGaN}-\mathrm{GaN}-\mathrm{AlGaN}$ electron blocking layer. $J$. Appl. Phys. 2012, 111, 094503-4.

(10) Ju, Z. G.; Tan, S. T.; Zhang, Z. H.; Ji, Y.; Kyaw, Z.; Dikme, Y.; Sun, X. W.; Demir, H. V. On the origin of the redshift in the emission wavelength of InGaN/GaN blue light emitting diodes grown with a higher temperature interlayer. Appl. Phys. Lett. 2012, 100, 123503-4.

(11) Zhang, L.; Ding, K.; Liu, N. X.; Wei, T. B.; Ji, X. L.; Ma, P.; Yan, J. C.; Wang, J. X.; Zeng, Y. P.; Li, J. M. Theoretical study of polarization-doped GaN-based light-emitting diodes. Appl. Phys. Lett. 2011, 98, 101110-3.

(12) Ji, Y.; Zhang, Z.-H.; Tan, S. T.; Ju, Z. G.; Kyaw, Z.; Hasanov, N.; Liu, W.; Sun, X. W.; Demir, H. V. Enhanced hole transport in InGaN/ GaN multiple quantum well light-emitting diodes with a p-type doped quantum barrier. Opt. Lett. 2013, 38, 202-204.

(13) Han, S. H.; Lee, D. Y.; Lee, S. J.; Cho, C. Y.; Kwon, M. K.; Lee, S. P.; Noh, D. Y.; Kim, D. J.; Kim, Y. C.; Park, S. J. Effect of electron blocking layer on efficiency droop in InGaN/GaN multiple quantum well light-emitting diodes. Appl. Phys. Lett. 2009, 94, 231123-3. 